\title{
Sonoanatomy revisited: the long thoracic nerve
}

\author{
Ke-Vin Chang ${ }^{1,2}$, Wei-Ting Wu ${ }^{1}$, Kamal Mezian ${ }^{3}$, Ondřej Naňka ${ }^{4}$, Levent Özçakar ${ }^{5}$
}

\begin{abstract}
${ }^{1}$ Department of Physical Medicine and Rehabilitation, National Taiwan University Hospital, Bei-Hu Branch, Taipei, Taiwan, ${ }^{2}$ Department of Physical Medicine and Rehabilitation, National Taiwan University, College of Medicine, Taipei, Taiwan, ${ }^{3}$ Department of Rehabilitation Medicine, Charles University, First Faculty of Medicine, Prague, Czech Republic, ${ }^{4}$ Institute of Anatomy, Charles University, First Faculty of Medicine, Prague, Czech Republic, ${ }^{5}$ Department of Physical and Rehabilitation Medicine, Hacettepe University Medical School, Ankara, Turkey
\end{abstract}

\begin{abstract}
The long thoracic nerve (LTN) arises from the C5, C6 and C7 roots and innervates the serratus anterior muscle. Scapular winging is the landmark manifestation of LTN neuropathy and may develop after an accidental injury during selective cervical nerve root or inter-scalene brachial plexus blocks. Although its sonoanatomy has been described in the previous literature, how to gradually identify the entire nerve course has rarely been discussed. In this regard, the present paper aims to elaborate the scanning method of the LTN from its origin to its distal segment.
\end{abstract}

Keywords: long thoracic nerve; anatomy; ultrasonography

\section{Introduction}

High resolution ultrasound has been widely used in imaging peripheral nerves and in the evaluation of brachial plexus lesions [1,2]. There are several nerves emerging from the root level of the brachial plexus, such as the dorsal scapular nerve (DSN) and long thoracic nerve (LTN) [1]. The DSN branches from the C5 nerve root, whereas the LTN arises from the C5, C6 and C7 roots. Both abovementioned nerves pierce the middle scalene muscles, making their courses so similar at the cervical level. The DSN innervates the levator scapulae

Received 17.03.2019 Accepted 10.04.2019

Med Ultrason

2019, Vol. 21, No 3, 349-352

Corresponding author: Ke-Vin Chang, $\mathrm{MD}, \mathrm{PhD}$

Department of Physical Medicine and

Rehabilitation, National Taiwan University

Hospital, Bei-Hu Branch, 87 Neijiang St,

Wanhua District, Taipei City 108, Taiwan

E-mail: kvchang011@gmail.com, pattap@pchome.com.tw and rhomboid muscles, whereas the LTN innervates the serratus anterior muscle. Since the LTN travels all the way from the neck to the truncal region, the long course renders it more vulnerable to injury. Scapular winging is the landmark manifestation of LTN neuropathy and may develop after an accidental injury during selective cervical nerve root or inter-scalene brachial plexus blocks [3]. Although its sonoanatomy has been described in the previous literature $[1,4]$, how to step-wisely identify the entire nerve course has rarely been discussed. In this regard, the present paper aims to elaborate the scanning method of the LTN from its origin to its distal segment.

\section{LTN at the cervical level}

The regional anatomy of the LTN at the cervical and supraclavicular levels is shown in fig 1 . The proximal portion of the LTN is formed by two branches; the upper and lower divisions. The former is usually derived from the $\mathrm{C} 5$ and $\mathrm{C} 6$ nerve roots, whereas the latter originates from the $\mathrm{C} 7$ nerve root [5]. Both divisions may conjoin inside or outside the middle scalene muscle. Since the 
upper division also gives off branches to innervate the upmost portion of the serratus anterior muscle, it would be more ideal to identify the lower division first if the investigator intends to track the whole LTN. In addition, the most proximal portion of the LTN may be difficult to visualize in some cases because of the small diameter and complicated branching pattern from its root origin. The abovementioned tip can prevent the investigator from misrecognizing the DSN (also arising from the C5 nerve root) as the LTN. In addition, the course of the DSN is more horizontal than that of the LTN. Therefore, the DSN is easier to visualize in its long axis while the transducer is placed at the horizontal plane of the neck. Another important point in order to differentiate the DSN from the LTN is to identify their exit through the middle scalene muscle. The DSN can be seen passing the fascial plane interposed by the levator scapulae and middle scalene muscles, whereas the exiting point of the LTN is covered by subcutaneous tissue only (Video 1, on the journal site).

Unlike the way that cervical nerve roots are scanned (where the transducer is placed along the horizontal plane to clarify the tubercles of the transverse process), the transducer should be positioned more obliquely for precisely visualizing the short axis of the middle scalene muscle. Once the $\mathrm{C} 7$ transverse process is seen, the posterior edge of the transducer is required to pivot cranially (fig 2). As the transducer has been placed obliquely, the cranial part of the brachial plexus may evolve to the trunk level but the caudal part of the brachial plexus still stays at the root (C7) level. The LTN can be seen following a nearly vertical course emerging from the middle scalene

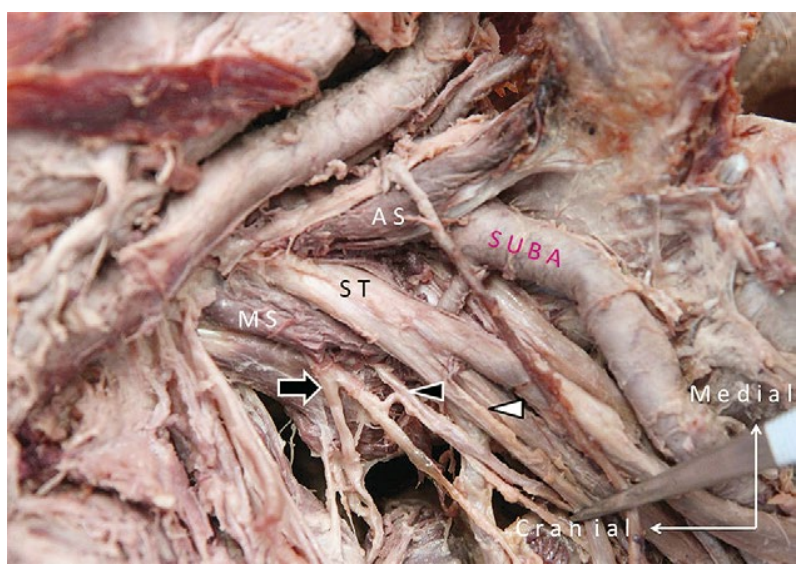

Fig 1. The cadaver model for the long thoracic nerve (black arrowhead) at the cervical and supraclavicular regions. White arrow head, suprascapular nerve; black arrow, dorsal scapular nerve; AS, anterior scalene muscle; MS, middle scalene muscle; SUBA, subclavian artery; ST, superior trunk of the brachial plexus.

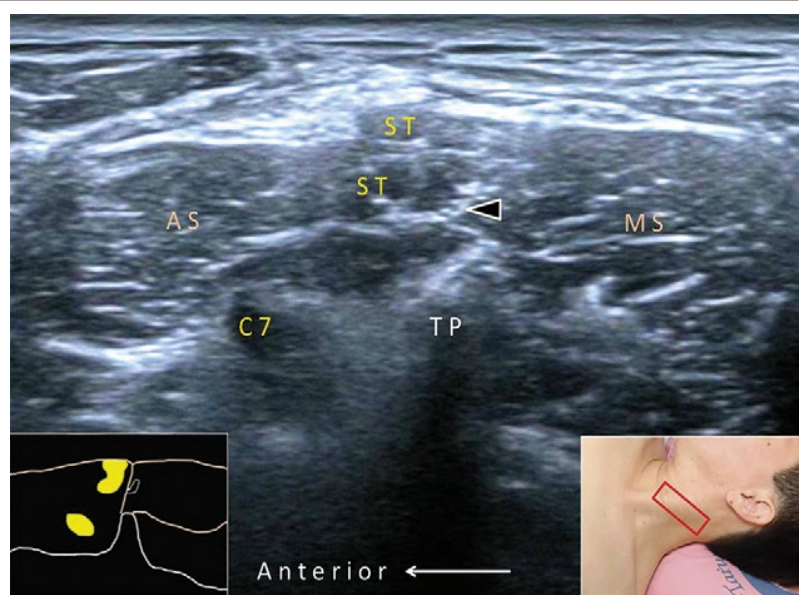

Fig 2. Ultrasound imaging of the long thoracic nerve (black arrowhead) at the cervical region. AS, anterior scalene muscle; MS, middle scalene muscle; ST, superior trunk of the brachial plexus. $\mathrm{C} 7$, the $7^{\text {th }}$ cervical nerve root; TP, posterior tubercle.

muscle. The investigator can slightly swap the posterior edge of the transducer if the LTN is not clearly visualized due to anisotropy. The swapping method is also helpful for identification of the root origins of the LTN which later converge into its main trunk.

\section{LTN at the supraclavicular level}

After the LTN exits the middle scalene muscle, it travels at the posterior aspect of the superior trunk of the brachial plexus. The reciprocal anatomy of the LTH (posterior) versus the brachial plexus (anterior) at the supraclavicular level is the same as that at the neck region. Herein, the suprascapular nerve, the first branch of

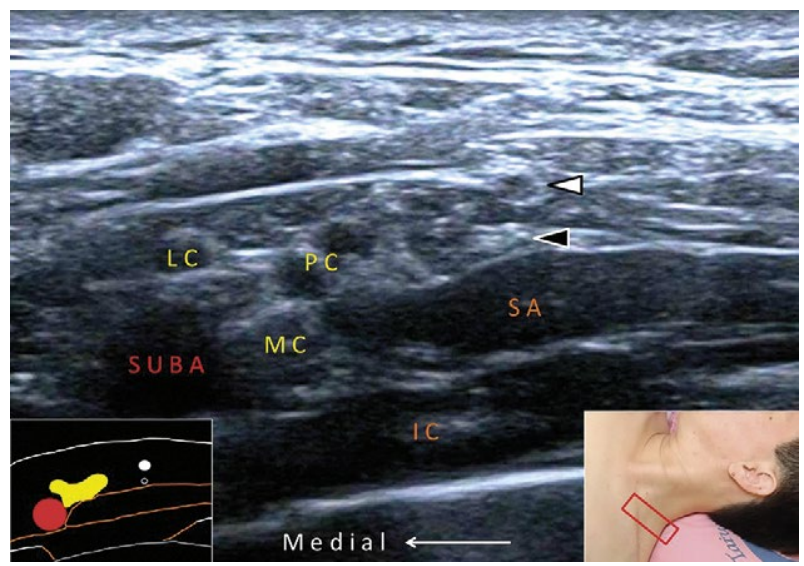

Fig 3. Ultrasound imaging of the long thoracic nerve (black arrowhead) at the supraclavicular region. White arrow head, suprascapular nerve; SUBA, subclavian artery; SA, serratus anterior muscle; LC, lateral cord of the brachial plexus; $\mathrm{MC}$, medial cord of the brachial plexus; PC, posterior cord of the brachial plexus; IC, intercostal muscle. 
the superior trunk, can be seen superficially to the LTN (fig 3). The size of the suprascapular nerve is usually larger than the LTN at this level [6]. The power Doppler mode can be used to recognize the transverse cervical or suprascapular arteries which pass above the LTN. Moving the transducer more laterally, the LTN is seen coursing on the superficial fascia of the serratus anterior muscle.

\section{LTN at the infraclavicular level}

Later, the LTN enters the costoclavicular space where the nerve is invisible due to the shadowing of the clavicle. The regional anatomy of the LTN at the infraclavicular and truncal level is shown in fig 4. After the LTN leaves the costoclavicular space, it can be seen again on top of the serratus anterior muscle posterior to the cord level of the brachial plexus (fig 5). At this point, the participant is required to fully abduct the shoulder to separate the LTN (towards the trunk) from the brachial plexus (towards the upper limb). Due to anisotropy, the LTN may be seen a little blurred here while it courses along the dome of the chest cage. Once the investigator notices that the border of the LTN becomes gradually obscure, the position of the transducer should be shifted from the ventral aspect of the anterior axillary fold to its dorsal aspect for better visualization of the LTN at the anterior border of the axillary fossa.

\section{LTN at the truncal level}

Finally, the LTN gradually travels toward the middle axillary line besides the lateral thoracic artery [4] (fig 6). Another structure that may be misrecognized as the LTN is the thoracodorsal nerve, which is located posterior to the LTN [4]. The tip to differentiate these two nerves is to

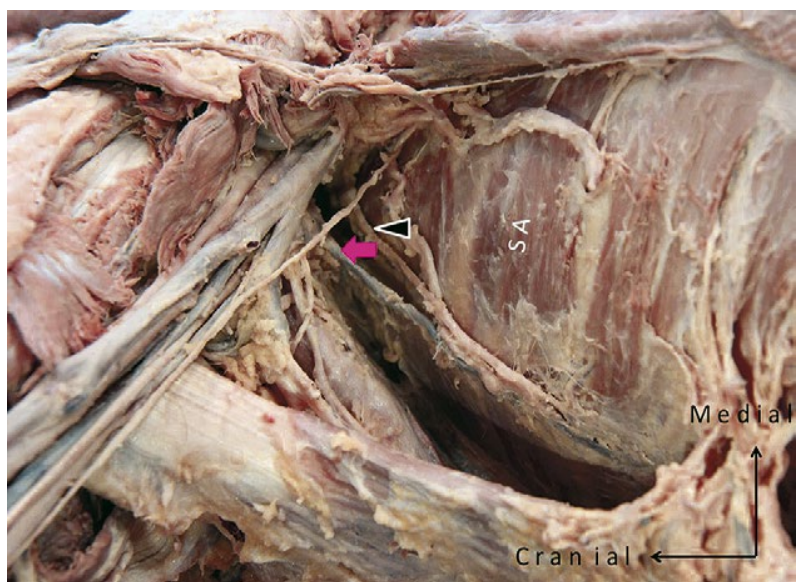

Fig 4. The cadaver model for the long thoracic nerve (black arrowhead) at the infraclavicular and truncal regions. SA, serratus anterior muscle; red arrow, lateral thoracic artery.

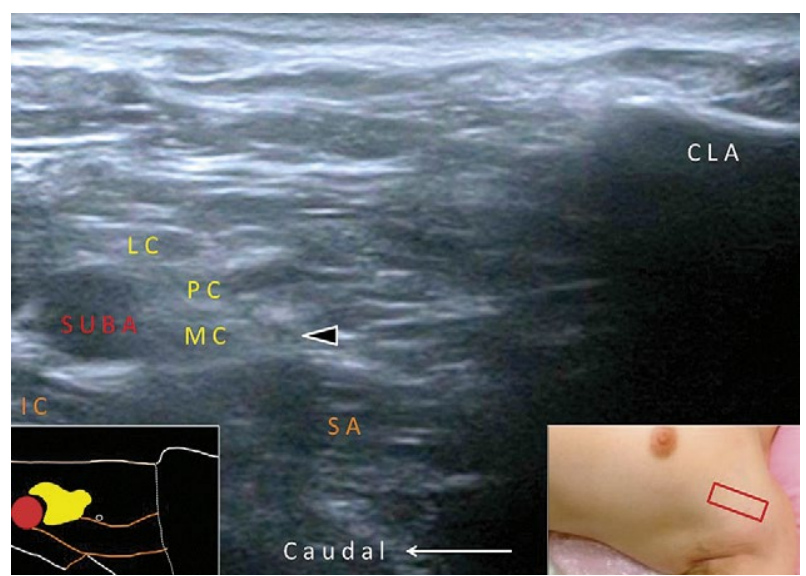

Fig 5. Ultrasound imaging of the long thoracic nerve (black arrowhead) at the infraclavicular region. SUBA, subclavian artery; SA, serratus anterior muscle; LC, lateral cord of the brachial plexus; MC, medial cord of the brachial plexus; PC, posterior cord of the brachial plexus; IC, intercostal muscle; CLA, clavicle

maximally abduct the axilla whereby the nerve coursing underneath (also innervating) the latissimus dorsi muscle would be the thoracodorsal nerve not the LTN. Furthermore, the LTN would be divided into several branches to innervate the serratus anterior muscle, which makes clear visualization of the LTN more challenging at its distal portion.

\section{Conclusion}

The present educational article elaborates the sonoanatomy of the LTN from its root origin to the distal end. With familiarization of its full course, visualization of the LTN by using a high-frequency linear transducer will



Fig 6. Ultrasound imaging of the long thoracic nerve (black arrowhead)at the truncalregion. SA, serratus anteriormuscle; IC, intercostal muscle; CLA, clavicle; red arrow, lateral thoracic artery. 
never be a tough task. However, the investigators should always keep in mind that variations of the nerves may exist on different participants. The transducer should be moved back and forth to confirm the continuity of the target nerves. Last but not least, the echotexture of the innervated muscle (serratus anterior) should be carefully inspected and compared with the contralateral site. In some cases with LTN neuropathy, the LTN may be difficult to locate but ultrasound imaging can easily reveal the associated atrophy of the serratus anterior muscle.

\section{References}

1. Chang KV, Lin CP, Hung CY, Ozcakar L, Wang TG, Chen WS. Sonographic Nerve Tracking in the Cervical Region: A Pictorial Essay and Video Demonstration. Am J Phys Med Rehabil 2016;95:862-870.
2. Chang KV, Mezian K, Nanka O, et al. Ultrasound Imaging for the Cutaneous Nerves of the Extremities and Relevant Entrapment Syndromes: From Anatomy to Clinical Implications. J Clin Med 2018;7:E457.

3. Chang KV, Wu WT, Ozcakar L. Ultrasound-Guided Interventions of the Cervical Spine and Nerves. Phys Med Rehabil Clin N Am 2018;29:93-103.

4. Chang KV, Lin CP, Lin CS, Wu WT, Karmakar MK, Ozcakar L. Sonographic tracking of trunk nerves: essential for ultrasound-guided pain management and research. J Pain Res 2017;10:79-88.

5. Bertelli JA, Ghizoni MF. Long thoracic nerve: anatomy and functional assessment. The J Bone Joint Surg Am 2005;87:993-998.

6. WuWT,ChangKV,MezianK,NankaO,LinCP,OzcakarL.Basis of Shoulder Nerve Entrapment Syndrome: An Ultrasonographic Study Exploring Factors Influencing Cross-Sectional Area of the Suprascapular Nerve. Front Neurol 2018;9:902. 